\title{
Parasitism of Locally Recruited Egg Parasitoids of the Fall Armyworm in Africa
}

\author{
Saidou A. Laminou ${ }^{1,2}$, Malick Niango Ba ${ }^{1, *}$ (D) , Laouali Karimoune ${ }^{1}$, Ali Doumma ${ }^{2}$ and \\ Rangaswamy Muniappan ${ }^{3}$ \\ 1 International Crops Research Institute for the Semi-Arid Tropics (ICRISAT), BP 12404 Niamey, Niger; \\ sayidelamine@gmail.com (S.A.L.); L.Karimoune@cgiar.org (L.K.) \\ 2 University Abdou Moumouni, BP 10896 Niamey, Niger; doumma@yahoo.com \\ 3 Virginia Tech, 526 Price Fork Road, Blacksburg, VA 24061-0378, USA; rmuni@vt.edu \\ * Correspondence: b.malick@cgiar.org; Tel.: +227-20315656
}

Received: 4 May 2020; Accepted: 24 June 2020; Published: 9 July 2020

\begin{abstract}
The fall armyworm (FAW), Spodoptera frugiperda (J.E. Smith) (Lepidoptera: Noctuidae), is an insect native to the tropical and subtropical Americas that has recently spread to Africa, where it predominately attacks maize, sorghum and other plant species. Biological control is an environmentally friendly way of combatting the pest and contributes to an integrated pest management approach. In Africa, several trichogrammatid parasitoids and Telenomus remus Nixon (Hymenoptera: Platygastridae) have been found parasitizing eggs of the FAW. In Niger, the egg parasitoids encountered include Trichogrammatoidea sp. (Hymenoptera: Trichogrammatidae) and Telenomus remus Nixon. Parasitism of the FAW eggs by the two egg parasitoids was assessed in the laboratory, followed by field testing on sentinel eggs. In the laboratory, T. remus parasitized on average $78 \%$ of FAW eggs, compared to $25 \%$ for Trichogrammatoidea sp. Telenomus remus was able to parasitize egg masses that were fully covered with scales, while Trichogrammatoidea sp. parasitized only uncovered egg masses. On-farm releases of T. remus in sorghum fields caused up to $64 \%$ of FAW egg parasitism. Parasitized eggs yielded viable progeny, which can contribute to FAW egg parasitism build-up during the cropping season. Our findings lay the groundwork for the use of T. remus in augmentative releases against FAW in Africa.
\end{abstract}

Keywords: Telenomus remus; augmentative release; biological control; trichogrammatid; Spodoptera frugiperda; fall armyworm; Niger; Sorghum

\section{Introduction}

The fall armyworm (FAW), Spodoptera frugiperda (J.E. Smith) (Lepidoptera: Noctuidae), is an insect native to the tropical and subtropical Americas that spread to Africa in early 2016 [1]. It became a serious pest mainly to maize, with early estimates in 2017 reporting yield losses of up $20.6 \mathrm{~m}$ tons in just 12 maize-producing countries [2]. Additionally, FAW attacks many other important food crops including sorghum and millet in Africa [3-6]. This damage has created problems all over Africa, leading to emergencies and responses by farmers with unproven efficacy, such as the application of ash, sand, botanical extracts, and other locally available materials [7]. Further, different governments procured synthetic pesticides, including highly toxic ones, and distributed them to the farmers $[7,8]$. To mitigate the risk of highly hazardous and broad-spectrum chemicals, alternate approaches are being developed, including plant-derived pesticides [9-11], habitat management practices through the use of companion cropping or push-pull technology [12,13], and biological control (BC) [14-16].

Biological control can offer an economical and environmentally friendly alternative for controlling FAW. In the Americas, BC is based on a variety of egg and larval parasitoids and predators [17-21]. 
The biological control approach contributes to an integrated pest management (IPM) strategy with other control means. As part of an IPM strategy, surveys of natural enemies of FAW were also conducted in several African countries [14-16,22,23]. These studies reported a wide range of larval parasitoids and egg parasitoids [14-16,22,23]. The egg parasitoid included Telenomus remus Nixon (Hymenoptera: Platygastridae) and several trichogrammatid species [15,16,22]. In Niger, the parasitoids encountered in maize and sorghum fields included several species belonging to the families Braconidae, Ichneumonidae, and Tachinidae, which parasitize larvae of FAW [24]. In addition, the two egg parasitoids Telenomus sp. and Trichogrammatoidea sp. were recorded [24]. Telenomus species was later identified as Telenomus remus Nixon [22]. Trichogrammatoidea sp. specimens ( $\mathrm{N}^{\circ} 1569$ NIGER Sadore 05-03-2019; NHMUK DNA21569/1 to NHMUK DNA21569/6) are permanently deposited in the Department of Life Sciences, Natural History Museum, London, UK [Polaszek A. prep]. T. remus is native to the Malay Peninsula and has been used in augmentative biological control (ABC) programs against $S$. frugiperda in the Americas [17,25-28]; however, since FAW and the parasitoid T. remus are being associated for the first time in Africa, it is necessary to assess the performance of this parasitoid before recommending its uses for ABC. Likewise, FAW and the parasitoid Trichogrammatoidea sp. are being associated for the first time; it is also worth assessing its performance while awaiting species identification. The first objective was to compare parasitism rates of FAW eggs between T. remus and Trichogrammatoidea sp. in the laboratory. Additionally, given that egg masses laid by FAW females are covered with scales from the female abdomen [25], we checked whether this feature affected parasitism by the two parasitoid species. The second objective was to test under field conditions, using sorghum plants infested with FAW egg masses, the parasitoid found to have a higher parasitism rate in the laboratory trials.

\section{Materials and Methods}

\subsection{Study Environment}

The lab bioassays and insect cultures were carried out in the entomology laboratory of the International Crops Research Institute for the Semi-Arid Tropics (ICRISAT) at Sadore in Niger (latitude $13^{\circ} 15^{\prime} \mathrm{N}$, longitude $2^{\circ} 18^{\prime} \mathrm{E}$ ) under a mean temperature of $25.60 \pm 0.02^{\circ} \mathrm{C}$ and mean relative humidity of $52.90 \pm 0.16 \%$. The on-station field releases experiment was conducted at the research campus of ICRISAT, Sadore. This agroecosystem has a unimodal rainfall pattern, with the rainy season extending from mid-May to mid-October. The field tests were carried out first in September 2019 toward the end of the rainy season and repeated in January 2020 after the rainy season. The average field temperature and relative humidity during FAW egg exposure and parasitoid releases were $31.23 \pm 0.36{ }^{\circ} \mathrm{C}$ and $65 \pm 1.75 \%$, respectively, for the first field test. The average field temperature and relative humidity were $19.69 \pm 0.10^{\circ} \mathrm{C}$ and $17.48 \pm 0.57 \%$, respectively, for the second field test.

\subsection{Insect Cultures for Bioassays}

The insects mass-reared for this study included the FAW, S. frugiperda, the rice moth, Corcyra cephalonica Stainton (Lepidoptera: Pyralidae), and the parasitoid wasps, T. remus and Trichogrammatoidea sp.

A colony of FAW was started from larvae collected in a sorghum field in late 2017 at the ICRISAT Research Station in Sadore, Niger. The insects were routinely reared in the laboratory. First instar larvae were fed with the tobacco budworm diet (Product\# F9781B) from Frontier ${ }^{\mathrm{TM}}$ Agricultural Sciences, Newark, DE, USA. From second instars on, the larvae were exclusively fed on leaves of castor bean (Ricinus communis L.) using the method described by Prasanna et al. [29]. The FAW larvae were supplied fresh castor leaf, which was cut into small pieces. Three FAW larvae were put together in $36.96 \mathrm{ml}$ plastic vials and supplied with $4.3 \mathrm{~g}$ of castor leaves. The leaves were replaced every $2-3$ days depending on how long they remained green and fresh. The FAW larvae on the natural diet usually matured after 15-20 days, with a pupation period of 10 days. Moths of the FAW were given fresh leaves of sorghum to lay eggs in oviposition wire mesh cages $(30 \times 30 \times 30 \mathrm{~cm})$. Cotton wool soaked 
with sugar (10\% sucrose in water solution) was hung in the cages to feed the moths. The leaves with attached eggs were cut and placed in egg hatching boxes. In about 4-6 days, the eggs developed into a blackhead stage and then hatched into neonate larvae. The neonate larvae were used to infest the artificial diet for the continuation of the colony. New wild FAW caterpillars were added to the colony in 2018 and 2019 to maintain insect vigor.

A colony of the rice moth C. cephalonica was established in the laboratory at ICRISAT, Sadore from wild insects collected in farmers' granaries in Niger in 2015. The insects were routinely reared on a mixture of pearl millet grain and flour in plastic buckets at an ambient temperature [30]. Usually, adults emerged after one month. New wild adults were added to the colony every year to maintain insect vigor.

A colony of Trichogrammatoidea sp. was initially started from field-collected eggs of FAW in a sorghum field on the ICRISAT campus, Sadore in late 2018. The colony was maintained using the method described for the mass-rearing of related species Trichogrammatoidea armigera Nagaraja (Hymenoptera: Trichogrammatidae) [31]. Eggs were kept in Petri dishes in the laboratory under the above-mentioned temperature and humidity conditions until the emergence of adults. Emerging Trichogrammatoidea were collected daily, sexed, and placed in plastic tubes $(\varnothing=2 \mathrm{~cm}, \mathrm{~h}=5.25 \mathrm{~cm})$. The eggs of $C$. cephalonica were used to rear the parasitoid Trichogrammatoidea sp. The use of $C$. cephalonica eggs for rearing Trichogrammatoidea sp. was supported by early findings suggesting that Trichogramma ability to parasitize $S$. frugiperda was not affected once reared on related Ephestia kuehniella Zeller species $[21,26,27]$. The eggs of $C$. cephalonica were irradiated to halt their development and avoid the cannibalization of eggs by hatched larvae from unparasitized eggs as described earlier [31]. Irradiation of eggs was performed in a dark chamber under UV light $4 \mathrm{~W}$ tube (UVP, USA, $254 \mathrm{~nm}$ ) for $45 \mathrm{~min}$ at a distance of $3 \mathrm{~cm}$. C. cephalonica eggs were glued on white rectangular cards $(4.5 \mathrm{~cm} \times 1.75 \mathrm{~cm})$, and a drop of honey was placed at the corner of the card to feed adult parasitoids. The culture was routinely maintained by the exposure of a first set of 30 eggs (1 day old) of C. cephalonica to a mated Trichogrammatoidea sp. female; this was followed by subsequent sets of 30 eggs ( 1 day old) every day until the death of the female. The females were provided a new male for mating every 3 days for $24 \mathrm{~h}$. The average life span of Trichogrammatoidea sp. female was $11.34 \pm 1.26$ days; the development from egg to adult was completed in $9.65 \pm 0.57$ days. Each female produced approximately 120 adults in a female biased sex-ratio 1:2.

The rearing technique of T. remus is similar to Trichogrammatoidea sp. except FAW eggs were used instead of $C$. cephalonica. The colony of $T$. remus was initially started from field-collected parasitized eggs of FAW in a sorghum field at ICRISAT campus, Sadore in late 2018. Emerging adults were sexed and given egg masses of FAW collected from the mass-rearing colony. Leaves of sorghum bearing FAW egg masses were cut and glued onto a carboard sheet $(7.3 \mathrm{~cm} \times 4.2 \mathrm{~cm})$, and a droplet of honey was placed at the corner of the card to feed T. remus adults. The egg cards were exposed for 2 days to a mated T. remus female in plastic flasks $(\varnothing=4.5 \mathrm{~cm} ; \mathrm{h}=11.5 \mathrm{~cm})$ using a ratio of 20 eggs to 1 wasp. The female was given fresh FAW egg masses every 2 days to parasitize until her death. The average life span of the T. remus female was approximately $17.5 \pm 2.21$ days; the development from egg to adult was completed in $11.21 \pm 0.63$ days. Each female produced approximately 200 adults in a female-biased sex-ratio.

\subsection{Assessment of FAW Egg Parasitism by T. remus and Trichogrammatoidea sp. in the Laboratory}

This experiment was conducted using the fresh egg masses of FAW collected from a mass-rearing facility. In general, eggs laid by FAW females were covered with scales from the female abdomen [25], and as the female aged, egg masses were less or not covered [32]. The egg masses were split into three groups: (i) egg masses fully covered with scales, (ii) egg masses partially covered with scales, and (iii) egg masses without scales. In our rearing condition, each of the egg masses had roughly 100 eggs. The different egg masses were exposed to 24-h-old mated females of T. remus, Trichogrammatoidea sp., or combined T. remus + Trichogrammatoidea sp. for $24 \mathrm{~h}$ to allow oviposition. The experiment was carried out in three-factor (Egg scale, type of parasitism; egg scale $\times$ parasitism type interaction) 
factorial with 3 levels within each factor, and with 13 replicates. For each treatment, we used 24-h-old mated wasp females. The incubation of eggs and parasitoids were carried out in plastic vials $(\varnothing=3 \mathrm{~cm}$; $\mathrm{h}=8.5 \mathrm{~cm}$ ) for $24 \mathrm{~h}$, and the parasitized eggs were incubated until the emergence of the new generation of parasitoids. Data on parasitism and emerging adults were recorded.

\subsection{Assessment of Field Performance of Telenomus remus Following Augmentative Releases}

\subsubsection{Preparation of the T. remus Cards for On-Station Field Releases}

All T. remus parasitoids used in the releases were from laboratory colonies reared on FAW eggs. Parasitoid cards $(7.3 \mathrm{~cm} \times 4.2 \mathrm{~cm}$ dimension) were prepared 11 days prior to field releases. Each card had 14 irradiated egg masses of FAW (with $\approx 50$ eggs in each) parasitized with 12 T. remus mated females for 48 h. T. remus adults began emerging from cards the day they were placed in the field, and after 2 days, all the parasitoids had emerged. Each card produced approximately 415 parasitoids (60\% females). The parasitoid cards were placed individually in a thick envelope to protect them from sunlight, direct rainfall, and predation. The envelope was perforated to create small holes that allowed emerging T. remus adults to disperse.

All T. remus parasitoids used in the releases were from laboratory colonies reared on FAW eggs. Parasitoid cards $(7.3 \mathrm{~cm} \times 4.2 \mathrm{~cm}$ dimension) were prepared 11 days prior to field releases. Each card had 14 irradiated egg masses of FAW (with $\approx 50$ eggs in each) parasitized with 12 T. remus mated females for 48 h. T. remus adults began emerging from cards the day they were placed in the field, and after 2 days, all the parasitoids had emerged. Each card produced approximately 415 parasitoids (60\% females). The parasitoid cards were placed individually in a thick envelope to protect them from sunlight, direct rainfall, and predation. The envelope was perforated to create small holes that allowed emerging T. remus adults to disperse.

\subsubsection{On-Station Releases}

This experiment was performed first in September 2019 and repeated in January 2020. The experiment was carried out on sorghum. For each of the two trials, a local popular sorghum variety named Hankorin karuwa (with an 85 day maturing cycle, sensitive to FAW) was planted in a spacing arrangement of $1 \mathrm{~m} \times 1 \mathrm{~m}$. For each trial, the sorghum was planted in eight fields of $200 \mathrm{~m}^{2}$ each with 400 sorghum plants at 200 stands (two plants/stand). All consecutive fields were separated by $200 \mathrm{~m}$ of grassland. The eight fields were divided into two groups of four: (i) four release fields that were each supplied with T. remus parasitoid, and (ii) four control fields that did not receive any parasitoids. Within each of the eight fields, one plant in every two stands was infested with FAW egg masses. This corresponds to 100 sorghum plants infested in each plot $(25 \%)$. The plants were infested 2 weeks after planting using the sentinel egg technique [20,33,34]. Freshly laid egg masses of FAW were removed from sorghum leaves in the laboratory culture, taken to the field, and pasted to the upper face of sorghum leaves with nontoxic glue to mimic naturally laid eggs. The egg masses were covered with a thin mesh $(\varnothing=1 \mathrm{~mm})$ that prevented the predation of eggs but allowed parasitism by parasitoids. Each selected plant was infested with one egg mass of $\approx 50$ eggs.

The infestation of sorghum with FAW eggs occurred on the same day as the placement of parasitoid cards. The parasitoids were released once at different points of the field using cards with parasitized FAW eggs on the day T. remus adults began emerging. The envelopes bearing the parasitoid cards were fastened to a sorghum stalk at $0.5 \mathrm{~m}$ above ground at three points of the field. All the parasitoids typically emerged in 2 days. No parasitoids were released in any of the control fields. The T. remus parasitoid was released at the rate of 15 T. remus females per $100 \mathrm{FAW}$ eggs as recommended in other settings [35]. This corresponded to 1250 parasitoids per $200 \mathrm{~m}^{2}$ (750 females and 500 males) and three parasitoid cards per release field. Egg masses that were placed in both release and control fields were recollected 4 days after exposure and taken back to the laboratory for incubation at ambient temperature 
and the observation of parasitism, emergence of parasitoids, and dead eggs. Emerging parasitoids were identified and counted.

\subsection{Data Analysis}

The percentage parasitism was computed by calculating the ratio of the total number of parasitized eggs to the total of eggs counted within an egg mass. The percentage of emerging parasitoids was calculated as the ratio of the total number of emerging adults to the total of parasitized eggs. The assumptions of normality of residuals was confirmed using the Shapiro-Wilk test. The data were subjected to arcsine transformation prior to ANOVA with SAS software version 9.1 [36]. Laboratory data were subjected to two-way ANOVA to compare parasitoid species (3 levels) and egg mass type (3 level) and their interactions. Likewise, field data of all years were combined together and subjected to two-way ANOVA to compare treatments and years and their interactions. Means were separated by Bonferroni post hoc test.

\section{Results}

\subsection{Parasitism of FAW Eggs by T. remus and Trichogrammatoidea sp. in the Laboratory}

The egg mass coverage significantly affects the parasitism regardless of species $\left(\mathrm{F}_{2,106}=5.67\right.$; $p=0.005)$. Likewise, the parasitism was significantly affected by parasitoid species regardless of egg mass type $\left(\mathrm{F}_{2,106}=74.07 ; p<0.001\right)$. However, the egg mass coverage and parasitoid species interaction does not affect the parasitism $\left(\mathrm{F}_{4,106}=2.18 ; p=0.07\right)$. The parasitism on uncovered egg masses was significantly higher than the two other egg masses (Figure 1). The egg parasitism by T. remus was significantly higher than the parasitism by Trichogrammatoidea sp. and combined T. remus + Trichogrammatoidea sp. regardless of egg mass type (Figure 1).

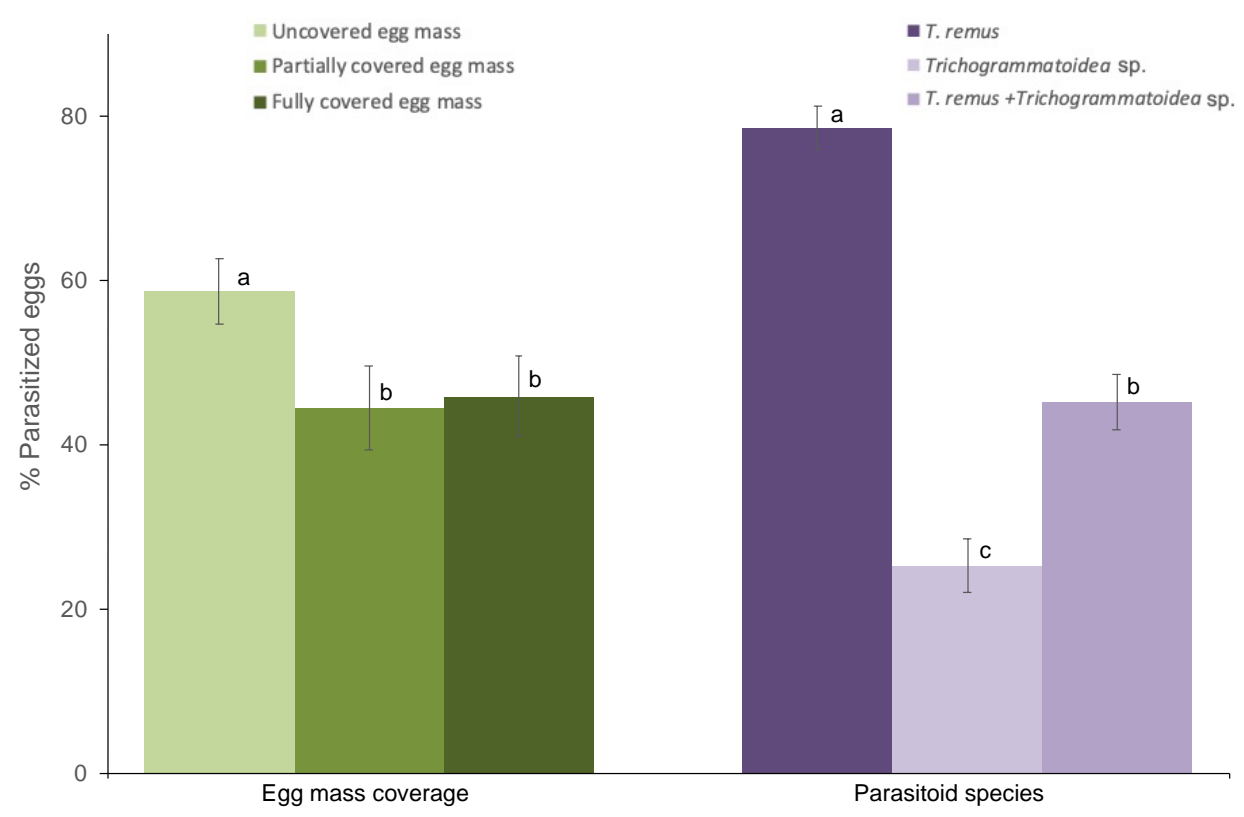

Figure 1. Egg parasitism of Spodoptera frugiperda (\% $\pm \mathrm{SE})$ by T. remus, Trichogrammatoidea sp.; and combined T. remus + Trichogrammatoidea sp. of fully covered, partially covered, and uncovered egg masses. Bars bearing different letters within column groups were significantly different (Bonferroni test, $\alpha=0.05)$.

The egg mass coverage does not affect parasitoid emergence regardless of species $\left(\mathrm{F}_{2,102}=1.55\right.$; $p=0.21$; Figure 2). However, progeny emergence varied by parasitoid species $\left(\mathrm{F}_{2,102}=84.37\right.$; $p<0.001)$. The interaction between parasitoid species and egg mass coverage does not affect emergence 
$\left(\mathrm{F}_{4,102}=1.04 ; p=0.39\right)$. Eggs subjected to T. remus parasitism yielded significantly more progeny than combined T. remus + Trichogrammatoidea sp. or Trichogrammatoidea sp. alone regardless of egg mass type (Figure 2). Emerging progeny from combined parasitism included T. remus (97\%) and Trichogrammatoidea sp. (3\%).

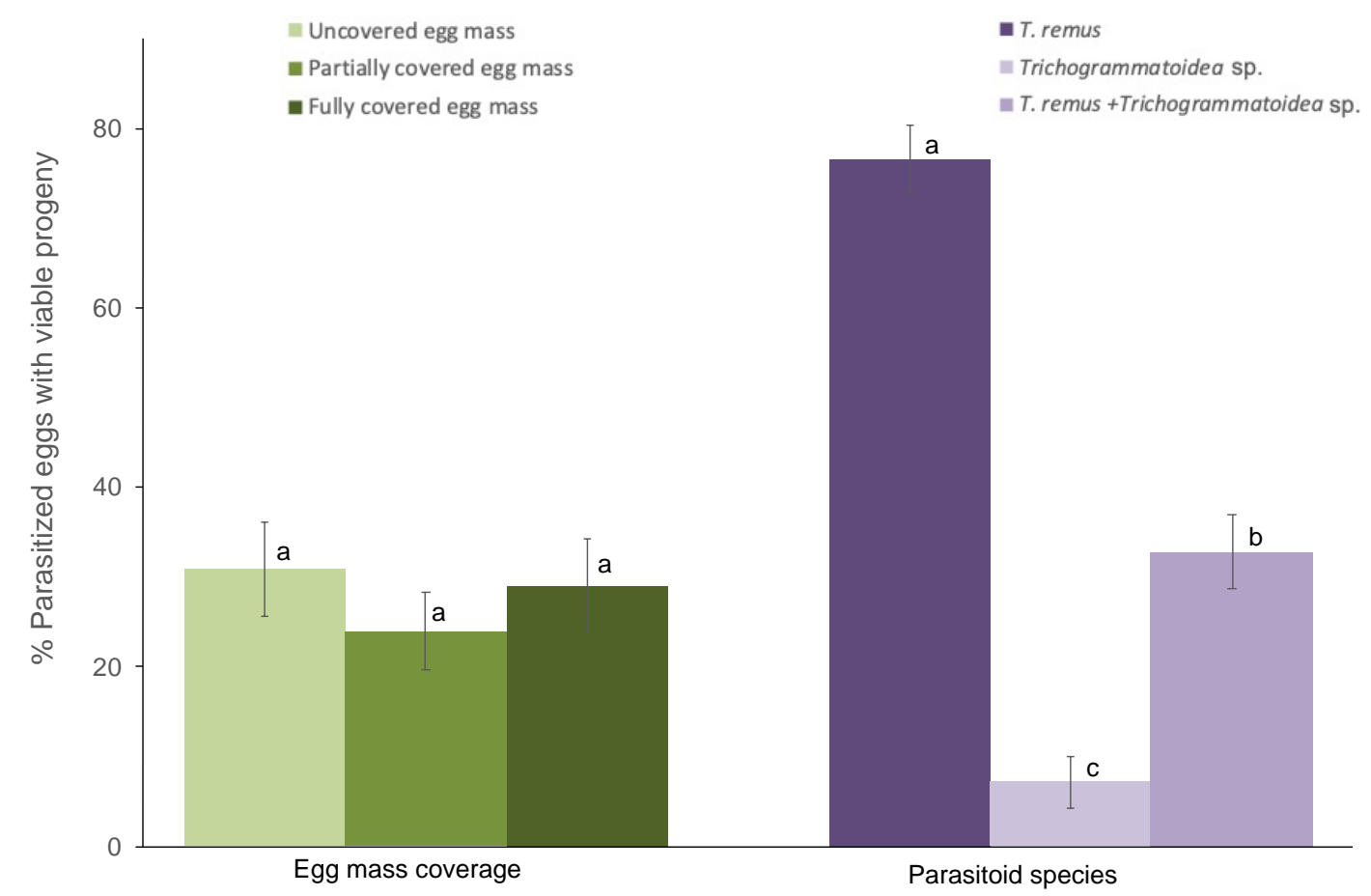

Figure 2. Viable progeny emerging from eggs of Spodoptera frugiperda $(\% \pm \mathrm{SE})$ parasitized by T. remus, Trichogrammatoidea sp., and combined T. remus + Trichogrammatoidea sp. of fully covered, partially covered, and uncovered egg masses. Bars bearing different letters within column groups were significantly different (Bonferroni test, $\alpha=0.05$ ).

\subsection{Parasitism of T. remus Following on-Station Releases}

The overall parasitism for both trials was significantly higher in released fields than control fields $\left(F_{1,12}=93.99 ; p<0.001 ;\right.$ Figure 3$)$. However, a significant difference was not found for years $\left(F_{1,12}=1.59\right.$; $p=0.23)$. Likewise, the years and treatments interaction does not affect the parasitism $\left(\mathrm{F}_{1,12}=0.29\right.$; $p=0.60)$.

For both trials, up to $77 \%$ parasitized eggs yielded viable $T$. remus progeny, with no significant difference between released and control fields $\left(\mathrm{F}_{1,10}=1.01 ; p=0.34\right.$; Figure 3). Likewise, a significant difference was not found for years $\left(\mathrm{F}_{1,10}=0.04 ; p=0.85\right)$. However, the years and treatments interaction affect parasitoids emergence $\left(\mathrm{F}_{1,10}=7.32 ; p=0.02\right)$. Emerging progeny included $\mathrm{T}$. remus $(95 \%)$ and Trichogrammatoidea sp. (5\%) in both released and control fields in September 2019, while in the January 2020 trial, only T. remus progeny emerged from parasitized eggs of both treatments. 


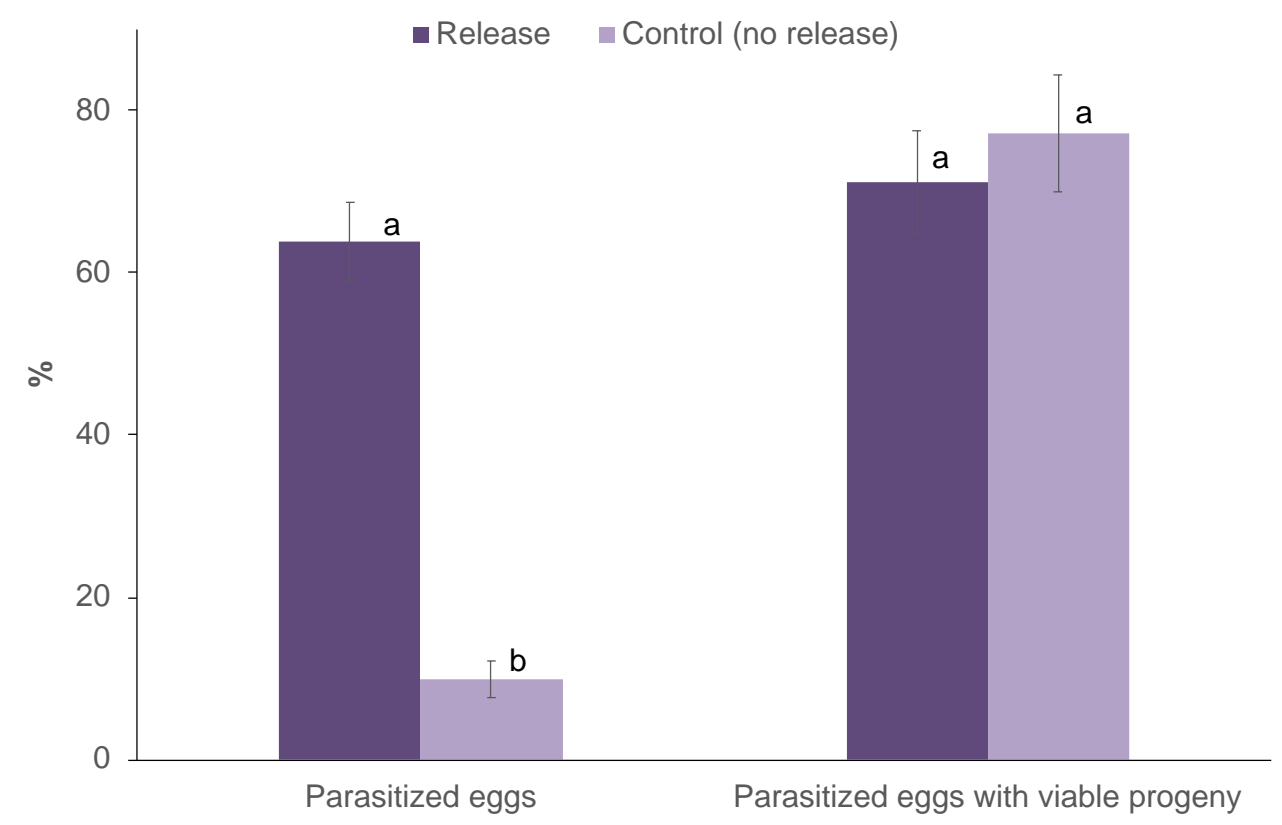

Figure 3. Parasitized eggs of Spodoptera frugiperda (\% \pm S.E.) in release and control fields following on-station augmentative releases of $T$. remus and corresponding emerging progenies ( $\% \pm$ S.E.). For each parameter, treatments bearing different letters were significantly different (Bonferroni test, $\alpha=0.05$ ).

\section{Discussion}

In the laboratory, T. remus parasitized significantly more FAW eggs than Trichogrammatoidea sp. Unlike Trichogrammatoidea sp., T. remus was able to overcome the layer of scales on egg masses and parasitize all types of egg masses equally. This is consistent with previous findings [37,38]. T. remus was described as an aggressive parasitoid due to its larger, more robust size (as compared to Trichogrammatoidea), which enables it to penetrate all layers of the egg mass of FAW and parasitize more eggs [25]. Similar observations were made for the related species Telenomus nawai Ashmead on the eggs of related host species Spodoptera litura Fabricius [39]. In our experimental conditions, T. remus parasitized on average 78\% of FAW eggs in the laboratory. This is below the 100\% FAW egg parasitism reported in other settings for $T$. remus $[25,37]$. The discrepancies may be due to laboratory conditions, as the performance of T. remus is affected by temperature and humidity [40-43]. Differences may also be explained by parasitoid strains [35], as well as the egg mass/parasitoid ratio [44] and exposure time.

Usually, the presence of scales over FAW egg masses constitutes a barrier against parasitism by Trichogrammatidae $[45,46]$. It is thus not surprising that Trichogrammatoidea sp. did not parasitize more than $25 \%$ of FAW eggs, with most parasitized eggs being those that were not covered by the protective scale. However, this level of parasitism by Trichogrammatoidea sp. is very low compared to Trichogramma pretiosum Riley (Hymenoptera: Trichogrammatidae), a parasitoid used in the biological control of FAW in Nicaragua [21]. In fact, T. pretiosum also avoids egg masses with too many scales, parasitizing only the upper layer of egg masses and those found on the edge of the egg masses, which can be easily reached from the side [47]. The poor performance of Trichogrammatoidea sp. on S. frugiperda could be due to an early learning experience as the parasitoid was mass reared on C. cephalonica before being given S. frugiperda eggs. Similar behavior has been reported with some Trichogramma species in other settings [48-50]. The poor performance of Trichogrammatoidea sp. on S. frugiperda could also be due to the size of the rearing eggs. In fact, eggs of C. cephalonica are smaller than those of $S$. frugiperda [51], and as reported in other settings, once reared in small eggs trichogrammatid could be smaller and this could affect the parasitism [52-56]. Further studies are, however, needed to reach conclusions as with T. pretiosum, its mass rearing on Ephestia kuehniella eggs does not affect its performance once releases in the field against FAW [21]. 
Given that T. remus emergence always occurred at the range of one parasitoid per host egg [25,40,57], and that $T$. remus parasitism was higher than that of Trichogrammatoidea sp., a higher emergence of $T$. remus adults when both parasitoids were put together in the same arena could have been expected. Unfortunately, the contrary was observed, meaning that the combination of T. remus and Trichogrammatidae sp. adversely affected the overall parasitism of FAW eggs. In fact, the interaction of different parasitoid species can lead to contrasting results [58-62]. This could be due to exploitative competition toward eggs of FAW and/or interference competition, as observed between Trichogramma pretiosum Riley, Trichogramma atopovirilia Oatman \& Platner and T. remus [34]. In fact, by being quicker in ovipositing and searching for FAW eggs, T. remus likely parasitized a larger number of eggs within a shorter period of time than its competitor [47], and thus subjected itself to superparasitism by Trichogrammatoidea sp., as suggested by previous studies [34]. Similar findings were observed for T. pretiosum on the related Telenomus heliothidis Ashmead species [63]. Further studies are, however, needed to reach conclusions on Trichogrammatoidea sp. and T. remus interactions. Although overall parasitism is affected, significantly higher numbers of T. remus emerged from parasitized eggs than Trichogrammatoidea sp. This reflects a competitive dominance of T. remus over Trichogrammatoidea sp. Similar findings have been reported for T. remus over Trichogramma atoporivilia and Trichogramma pretiosum [34,47], and for related Telenomus ullyetti Nixon over Trichogrammatoidea lutea Girault [64].

From the field experiments, releases of T. remus led to significantly higher parasitism of FAW eggs compared to control fields that did not receive parasitoids. Nevertheless, our study showed positive behavior characteristics for T. remus as a biological control agent of FAW in a sorghum cropping system. Interestingly, released T. remus were able to find and parasitize sentinel host eggs within the sorghum field. Similar findings were reported with T. remus in corn cropping systems infested with FAW in the Americas [25,26,28,34,37]. In our case, the augmentative release of T. remus resulted in $64 \%$ parasitism compared to $60 \%-90 \%$ in Latin America $[25,26,37]$. Discrepancies in parasitism could be due to differences in the host egg/parasitoid ratio, as reported earlier [28,65]. Furthermore, we used artificially infested plants, while previous studies operated under naturally infested crops. As observed in other settings, sentinel eggs could dramatically underestimate actual rates of parasitism by several parasitoid species [66,67], including the related species, Telenomus podisi Ashmead [68]. In fact, T. remus respond to a combination of kairomones including those present in the secretion of the S. frugiperda female accessory gland [69] and the sex pheromone of S. frugiperda [70]. Moreover, volatiles emitted by plants attacked by FAW caterpillars in the canopy can serve indirectly as cues for locating inconspicuous host stages, such as eggs by T. remus as reported in other settings [71]. In our case, both plant volatiles and sexual pheromones might be missing as wild FAW females and crop damage were not observed in the sorghum field during the experiment. All these considerations in combination could explain the lower T. remus parasitism in our experimental conditions. Another factor that could influence T. remus parasitism is the host crop. Most of the studies into T. remus parasitism on FAW are from maize cropping systems, while we used sorghum. As reported earlier, the performance of $T$. remus is affected by its host crop $[65,72,73]$. In fact, for several parasitoid species, the performance is habitat-specific [74-78]. Other factors such as temperature and humidity could explain the differences in parasitism $[38,40,43,73,79]$.

The importance of parasitoid emergence after on-farm releases must also be considered because it can compromise the maintenance of the parasitoid in the field. This is particularly necessary in an environment in which farmers plant at different times in the season and FAW lays eggs over an extended period of time, and when several generations of FAW could develop in the cropping season. T. remus progeny emerging from the first batch of released parasitoids can eventually contribute to the control of subsequent generations of FAW. As indicated by Ferrer [26], T. remus parasitism increased over weeks. In our case, the emergence of T. remus adults from parasitized eggs in the field was low compared to other findings, and this could be due to temperature. As reported by Bueno et al. [40], suitable temperatures for higher viability are between $20-31{ }^{\circ} \mathrm{C}$. Above $35{ }^{\circ} \mathrm{C}$, no T. remus adults emerged from parasitized eggs. In our case, the parasitized eggs were exposed to variable field 
temperatures for four days, which could reach $38^{\circ} \mathrm{C}$ at some points of the day. Similarly, when taken back to the laboratory for incubation, parasitized eggs experienced ambient temperature, which was also fluctuating. All these factors in combination could explain the discrepancies in the percentage of emergences. Interestingly, Trichogrammatoidea sp. adults emerged from sentinel egg masses of FAW subjected to T. remus augmentative releases in September 2019. This indicates that FAW eggs were also parasitized by naturally occurring trichogrammatids. A similar observation was reported in Brazil [28], and the researchers there also found significantly more parasitism due to released T. remus than naturally occurring by trichogrammatids. However, in January 2020 in the dry season, no Trichogrammatoidea sp. emerged from parasitized eggs, suggesting a seasonal occurrence of the parasitoid. These findings confirm the natural occurrence of Trichogrammatoidea sp. on eggs of FAW in Niger [24]. In other regions of Africa, trichogrammatid egg parasitoids were only encountered from the Trichogramma genus $[15,16]$.

\section{Conclusions}

This study showed that augmentative releases of the egg parasitoid T. remus significantly increase the parasitism of FAW eggs in sorghum fields. This finding lays the groundwork for the use of T. remus in biocontrol programs in an integrated approach for controlling FAW in Africa. Further on-farm assessment is being conducted to identify the exact numbers needed per crop acreage in relation to T. remus dispersal. Likewise, the rearing of $T$. remus on an alternative host, Corcyra cephalonica Stainton, should be considered for cost-effective mass production, as suggested by Viera et al. [80].

Author Contributions: Conceptualization, M.N.B.; data curation, S.A.L.; formal analysis, L.K.; funding acquisition, M.N.B.; methodology, S.A.L., M.N.B. and L.K.; resources, M.N.B.; supervision, M.N.B. and A.D.; validation, M.N.B.; visualization, S.A.L. and L.K.; writing—original draft, M.N.B.; writing一review \& editing, M.N.B., L.K., A.D. and R.M. All authors have read and agreed to the published version of the manuscript.

Funding: This research was funded by the CGIAR Research Program on Grain Legumes and Dryland Cereals (GLDC-CRP).

Acknowledgments: The language editing of this manuscript by Sara Hendery is greatly appreciated.

Conflicts of Interest: The authors declare no conflict of interest.

\section{References}

1. Goergen, G.; Kumar, P.L.; Sankung, S.B.; Togola, A.; Tamò, M. First report of outbreaks of the fall armyworm Spodoptera frugiperda (JE Smith) (Lepidoptera, Noctuidae), a new alien invasive pest in West and Central Africa. PLoS ONE 2016, 11, e0165632. [CrossRef] [PubMed]

2. Day, R.; Abrahams, P.; Bateman, M.; Beale, T.; Clottey, V.; Cock, M.; Colmenarez, Y.; Corniani, N.; Early, R.; Godwin, J.; et al. Fall armyworm: Impacts and implications for Africa. Outlooks Pest Manag. 2017, 28, 196-201. [CrossRef]

3. Abrahams, P.; Bateman, M.; Beale, T.; Clottey, V.; Cock, M.; Colmenarez, Y.; Corniani, N.; Day, R.; Early, R.; Godwin, J.; et al. Fall Armyworm: Impacts and Implications for Africa; CABI Evidence Note (2); Report to DFID.; CABI: Wallingford, UK, 2017. Available online: https://www.cabi.org/ISC/FullTextPDF/2018/20187200428.pdf (accessed on 26 March 2020).

4. Maiga, I.; Ndiaye, M.; Gagare, S.; Oumarou, G.; Oumarou, S. La chenille d'automne Spodoptera frugiperda, nouveau ravageur du maïs en Afrique de l'Ouest, a atteint le Niger; Centre Régional AGRHYMET: Niamey, Niger, 2017. Available online: http://www.reca-niger.org/IMG/pdf/Bulletin_special_Chenille.pdf (accessed on 26 March 2020).

5. Hruska, J.A. Fall armyworm (Spodoptera frugiperda) management by smallholders. CAB Rev. 2019, 14, 1-11. [CrossRef]

6. CABI. Spodoptera frugiperda. In Invasive Species Compendium; CAB International: Wallingford, UK, 2020. Available online: www.cabi.org/isc (accessed on 2 April 2020). 
7. Kumela, T.; Simiyu, J.; Sisay, B.; Likhayo, P.; Mendesil, E.; Gohole, L.; Tefera, T. Farmers' knowledge, perceptions, and management practices of the new invasive pest, fall armyworm (Spodoptera frugiperda) in Ethiopia and Kenya. Int. J. Pest Manag. 2019, 65, 1-9. [CrossRef]

8. Prasanna, B.M.; Huesing, J.E.; Eddy, R.; Peschke, V.M. Fall Armyworm in Africa: A Guide for Integrated Pest Management, 1st ed.; CIMMYT: Mexico City, Mexico, 2018.

9. Sisay, B.; Tefera, T.; Wakgari, M.; Ayalew, G.; Mendesil, E. The efficacy of selected synthetic insecticides and botanicals against fall armyworm, Spodoptera frugiperda, in Maize. Insects 2019, 10, 45. [CrossRef] [PubMed]

10. Phambala, K.; Tembo, Y.; Kasambala, T.; Kabambe, V.H.; Stevenson, P.C.; Belmain, S.R. Bioactivity of common pesticidal plants on fall armyworm larvae (Spodoptera frugiperda). Plants 2020, 9, 112. [CrossRef]

11. Rioba, N.B.; Stevenson, P.C. Opportunities and scope for botanical extracts and products for the management of fall armyworm (Spodoptera frugiperda) for smallholders in Africa. Plants 2020, 9, 207. [CrossRef]

12. Hailu, G.; Niassy, S.; Khan, Z.R.; Ochatum, N.; Subramanian, S. Maize-legume intercropping and Push-pull for management of fall armyworm, stemborers and striga in Uganda. Agron. J. 2018, 110, 2513-2522. [CrossRef]

13. Midega, C.A.O.; Pittchar, J.; Pickett, J.A.; Hailu, G.; Khan, Z.R. A climate-adapted push-pull system effectively controls fall armyworm, Spodoptera frugiperda (J.E. Smith), in maize in East Africa. Crop Prot. 2018, 105, 10-15. [CrossRef]

14. Sisay, B.; Simiyu, J.; Malusi, P.; Likhayo, P.; Mendesil, E.; Elibariki, N.; Wakgari, M.; Ayalew, G.; Tefera, T. First report of the fall armyworm, Spodoptera frugiperda (Lepidoptera: Noctuidae), natural enemies from Africa. J. Appl. Entomol. 2018, 142, 800-804. [CrossRef]

15. Sisay, B.; Simiyu, J.; Mendesil, E.; Likhayo, P.; Ayalew, G.; Mohamed, S.; Subramanian, S.; Tefera, T. Fall Armyworm, Spodoptera frugiperda infestations in East Africa: Assessment of damage and parasitism. Insects 2019, 10, 195. [CrossRef] [PubMed]

16. Agboyi, L.K.; Goergen, G.; Beseh, P.; Mensah, S.A.; Clottey, V.A.; Glikpo, R.; Buddie, A.; Cafà, G.; Offord, L.; Day, R.; et al. Parasitoid complex of fall armyworm, Spodoptera frugiperda, in Ghana and Benin. Insects 2020, 11, 68. [CrossRef] [PubMed]

17. Bueno, V.H.P.; van Lenteren, J.C. The popularity of augmentative biological control in Latin America: History and state of affairs. In Proceedings of the First International Symposium on Biological Control of Arthropods, Honolulu, HI, USA, 14-18 January 2002; International Organization of Biological Control: Antibes, France, 2002; pp. 180-184.

18. Molina-Ochoa, J.; Carpenter, J.E.; Heinrichs, E.A.; Foster, J.E. Parasitoids and parasites of Spodoptera frugiperda (Lepidoptera: Noctuidae) in the Americas and Caribbean basin: An inventory. Fla. Entomol. 2003, 86, 254-289. [CrossRef]

19. Figueiredo, M.D.L.C.; Cruz, I.; da Silva, R.B.; Foster, J.E. Biological control with Trichogramma pretiosum increases organic maize productivity by 19.4\%. Agron. Sustain. Dev. 2015, 35, 1175-1183. [CrossRef]

20. Jaraleño-Teniente, J.; Lomeli-Flores, J.R.; Rodríguez-Leyva, E.; Bujanos-Muñiz, R.; Rodríguez-Rodríguez, S.E. Egg parasitoids survey of Spodoptera frugiperda (Smith) (Lepidoptera: Noctuidae) in maize and sorghum in Central Mexico. Insects 2020, 11, 157. [CrossRef]

21. Castillo, P. Biological control in Nicaragua. In Biological Control in Latin America and the Caribbean: Its Rich History and Bright Future; van Lenteren, J.C., Bueno, V.H.P., Luna, M.G., Colmenarez, Y., Eds.; CAB International: Boston, MA, USA, 2020; pp. 336-344.

22. Kenis, M.; du Plessis, H.; Van den Berg, J.; Ba, M.N.; Goergen, G.; Kwadjo, K.E.; Baoua, I.; Buddie, A.; Cafà, G.; Offord, L.; et al. Telenomus remus, a candidate parasitoid for the biological control of Spodoptera frugiperda in Africa, is already present on the continent. Insects 2019, 10, 92. [CrossRef]

23. Koffi, D.; Kyerematen, R.; Eziah, V.Y.; Agboka, K.; Adom, M.; Goergen, G.; Meagher, R.L., Jr. Natural enemies of the fall armyworm, Spodoptera frugiperda (J.E. Smith) (Lepidoptera: Noctuidae) in Ghana. Fla. Entomol. 2020, 103, 85-90. [CrossRef]

24. Amadou, L.; Baoua, I.; Ba, M.N.; Karimoune, L.; Muniappan, R. Native parasitoids recruited by the invaded fall armyworm in Niger. Indian J. Entomol. 2018, 80, 1253-1254. [CrossRef]

25. Cave, R.D. Biology, ecology and use in pest management of Telenomus remus. Biocontrol News Inf. 2000, 21, 21-26.

26. Ferrer, F. Biological of agricultural insect pest in Venezuela: Advances, achievements, and future perspectives. Biocontrol News Inf. 2001, 22, 67-74. 
27. Parra, J.R.P. Egg parasitoids commercialization in the New World. In Egg Parasitoids in Agroecosystems with Emphasis on Trichogramma; Cônsoli, F.L., Parra, J.R.P., Zucchi, R.A., Eds.; Springer: New York, NY, USA, 2010; pp. 373-388.

28. Figueiredo, M.D.L.C.; Della Lucia, T.M.C.; Cruz, I. Effect of Telenomus remus Nixon (Hymenoptera: Scelionidae) density on control of Spodoptera frugiperda (Smith) (Lepidoptera: Noctuidae) egg masses upon release in a maize field. Rev. Bras. Milho Sorgo 2002, 1, 12-19. [CrossRef]

29. Prasanna, B.M.; Bruce, A.; Winter, S.; Otim, M.; Asea, G.; Sevgan, S.; Ba, M. Host plant resistance to fall armyworm. In Fall Armyworm in Africa: A Guide for Integrated Pest Management, 1st ed.; Prasanna, B.M., Huesing, J.E., Eddy, R., Peschke, V.M., Eds.; CIMMYT: Mexico City, Mexico, 2018; pp. 45-62.

30. Ba, M.N.; Baoua, I.B.; Kaboré, A.; Amadou, L.; Oumarou, N.; Dabire-Binso, C.; Sanon, A. Augmentative on-farm delivery methods for the parasitoid Habrobracon hebetor Say (Hymenoptera: Braconidae) to control the millet head miner Heliocheilus albipunctella (de Joannis) (Lepidoptera: Noctuidae) in Burkina Faso and Niger. BioControl 2014, 59, 689-696. [CrossRef]

31. Karimoune, L.; Ba, N.M.; Baoua, I.B.; Muniappan, R. The parasitoid Trichogrammatoidea armigera Nagaraja (Hymenoptera: Trichogrammatidae) is a potential candidate for biological control of the millet head miner Heliocheilus albipunctella (de Joannis) (Lepidoptera: Noctuidae) in the Sahel. Biol. Control 2018, 127, 9-16. [CrossRef]

32. Tefera, T.; Goftishu, M.; Ba, M.; Muniappan, R. A Guide to Biological Control of Fall Armyworm in Africa Using Egg Parasitoids, 1st ed.; ICIPE: Nairobi, Kenya, 2019. Available online: https://ipmil.cired.vt.edu/wp-content/ uploads/2019/10/A-Guide-to-Biological-Control-of-FAW_Final-updated.pdf (accessed on 27 March 2020).

33. Karimoune, L.; Ba, N.M.; Baoua, I.B.; Muniappan, R. Field performance of the parasitoid wasp, Trichogrammatoidea armigera (Hymenoptera: Trichogrammatidae) following releases against the millet head miner, Heliocheilus albipunctella (Lepidoptera: Noctuidae) in the Sahel. BioControl 2020, 65. [CrossRef]

34. Da Silva, C.S.B.; Vieira, J.M.; Loiácono, M.; Margaría, C.; Parra, J.R.P. Evidence of exploitative competition among egg parasitoids of Spodoptera frugiperda (Lepidoptera: Noctuidae) in maize. Rev. Colomb. Entomol. 2015, 41, 184-186.

35. Queiroz, A.P.; Bueno, A.F.; Pomari-Fernandes, A.; Bortolotto, O.C.; Mikami, A.Y.; Olive, L. Influence of host preference, mating, and release density on the parasitism of Telenomus remus (Nixon) (Hymenoptera, Platygastridae). Rev. Bras. Entomol. 2017, 61, 86-90. [CrossRef]

36. SAS. SAS version 9.1 for Windows; SAS Institute: Cary, NC, USA, 2003.

37. Cave, R.D.; Acosta, N.M. Telenomus remus Nixon: Un parasitoide en el control biológico del gusano cogollero, Spodoptera frugiperda (Smith). Ceiba 1999, 40, 215-227.

38. Pomari, A.F.; Bueno, A.D.F.; Bueno, R.C.O.D.F.; Menezes, A.D.O. Biological characteristics and thermal requirements of the biological control agent Telenomus remus (Hymenoptera: Platygastridae) reared on eggs of different species of the genus Spodoptera (Lepidoptera: Noctuidae). Ann. Entomol. Soc. Am. 2012, 105, 73-81. [CrossRef]

39. Fukuda, T.; Wakamura, S.; Arakaki, N.; Yamagishi, K. Parasitism, development and adult longevity of the egg parasitoid Telenomus nawai (Hymenoptera: Scelionidae) on the eggs of Spodoptera litura (Lepidoptera: Noctuidae). Bull Entomol. Res. 2007, 97, 185-219. [CrossRef]

40. Bueno, R.C.O.D.F.; Carneiro, T.R.; Pratissoli, D.; Bueno, A.D.F.; Fernandes, O.A. Biology and thermal requirements of Telenomus remus reared on fall armyworm Spodoptera frugiperda eggs. Ciência Rural 2008, 38, 1-6. [CrossRef]

41. Bueno, R.C.O.D.F.; Carneiro, T.R.; Bueno, A.D.F.; Pratissoli, D.; Fernandes, O.A.; Vieira, S.S. Parasitism capacity of Telenomus remus Nixon (Hymenoptera: Scelionidae) on Spodoptera frugiperda (Smith) (Lepidoptera: Noctuidae) eggs. Braz. Arch. Biol. Technol. 2010, 53, 133-139. [CrossRef]

42. Bueno, R.C.O.D.F.; Bueno, A.D.F.; Xavier, M.F.D.C.; Carvalho, M.M. Telenomus remus (Hymenoptera: Platygastridae) parasitism on eggs of Anticarsia gemmatalis (Lepidoptera: Eribidae) compared with its natural host Spodoptera frugiperda (Lepidoptera: Noctuidae). Ann. Entomol. Soc. Am. 2014, 107, 799-808. [CrossRef]

43. Pomari-Fernandes, A.; Queiroz, A.D.P.; Bueno, A.D.F.; Sanzovo, A.W.; Bortoli, S.D.A. The importance of relative humidity for Telenomus remus (Hymenoptera: Platygastridae) parasitism and development on Corcyra cephalonica (Lepidoptera: Pyralidae) and Spodoptera frugiperda (Lepidoptera: Noctuidae) eggs. Ann. Entomol. Soc. Am. 2015, 108, 11-17. [CrossRef] 
44. Morales, J.S.; Gallardo, J.S.V.; Vásquez, C.; Ríos, Y. Respuesta funcional de Telenomus remus (Hymenoptera: Scelionidae) a los huevos de Spodoptera frugiperda (Lepidoptera: Noctuidae). Bioagro 2001, 13, 49-55.

45. Beserra, E.B.; Parra, J.R.P. Impact of the number of Spodoptera frugiperda egg layers on parasitism by Trichogramma atopovirilia. Scientia Agricola 2005, 62, 190-193. [CrossRef]

46. Goulart, M.M.P.; Bueno, A.D.F.; Bueno, R.C.O.D.F.; Vieira, S.S. Interaction between Telenomus remus and Trichogramma pretiosum in the management of Spodoptera spp. Rev. Bras. Entomol. 2011, 55, 121-124. [CrossRef]

47. Carneiro, T.R.; Odair, A.; Fernandes, O.A. Interspecific interaction between Telenomus remus (Hymenoptera: Platygastridae) and Trichogramma pretiosum (Hymenoptera: Trichogrammatidae) on Spodoptera frugiperda (Lepidoptera: Noctuidae) eggs. Anais da Academia Brasileira de Ciências 2012, 84, 1127-1135. [CrossRef]

48. Kaiser, L.; Pham-Delegue, M.H.; Masson, C. Behavioural study of plasticity in host preferences of Trichogramma maidis (Hymenoptera: Trichogrammatidae). Physiol. Entomol. 1989, 14, 53-60. [CrossRef]

49. Supoyo, N.; Cribb, B.W.; Gordh, G. Experience acquisition by Trichogramma australicum Girault (Hymenoptera: Trichogrammatidae). Aust. Entomol. 1999, 38, 115-119.

50. Giunti, G.; Canale, A.; Messing, R.H.; Donati, E.; Stefanini, C.; Michaud, J.P.; Benelli, G. Parasitoid learning: Current knowledge and implications for biological control. Biol. Control 2015, 90, 208-219. [CrossRef]

51. Cônsoli, F.L.; Kitajima, E.W.; Parra, J.R.P. Ultrastructure of the natural and factitious host eggs of Trichogramma galho Zucchi and Trichogramma pretiosum Riley (Hymenoptera: Trichogrammatidae). Int. J. Insect Morphol. Embryol. 1999, 28, 211-231. [CrossRef]

52. Bigler, F.; Meyer, A.; Bosshart, S. Quality assessment in Trichogramma maidis Pintureau et Voegelé reared from eggs of the factitious hosts Ephestia kuehniella Zell. and Sitotroga cerealella (Olivier). J. Appl. Entomol. 1987, 104, 340-353. [CrossRef]

53. Van Bergeijk, K.E.; Bigler, F.; Kaashoek, N.K.; Pak, G.A. Changes in host acceptance and host suitability as an effect of rearing Trichogramma maidis on a factitious host. Entomol. Exp. Appl. 1989, 52, 229-238. [CrossRef]

54. Bai, B.; Luck, R.F.; Forster, L.; Stephens, B.; Janssen, J.A.M. The effect of host size on quality attributes of the egg parasitoid, Trichogramma pretiosum. Entomol. Exp. Appl. 1992, 64, 37-48. [CrossRef]

55. Greenberg, S.M.; Norlund, D.A.; Wu, Z. Influence of rearing host on adult size and ovipositional behavior of mass produced female Trichogramma minutum Riley and Trichogramma pretiosum Riley (Hymenoptera: Trichogrammatidae). Biol. Control 1998, 11, 43-48. [CrossRef]

56. Honda, J.Y.; Luck, R.F. Interactions between host attributes and wasp size: A laboratory evaluation of Trichogramma platneri as an augmentative biological control agent for two avocado pests. Entomol. Exp. Appl. 2001, 100, 1-13. [CrossRef]

57. Gerling, D. The development biology of Telenomus remus Nixon (Hymenoptera: Scelionidae). Bull. Entomol. Res. 1972, 61, 385-388. [CrossRef]

58. Briggs, C.J.; Latto, J. Interactions between the egg and larval parasitoids of a gall forming midge and their impact on the host. Ecol. Entomol. 2001, 26, 109-116. [CrossRef]

59. Finke, D.L.; Denno, R.F. Predator diversity and the functioning of ecosystems: The role of intraguild predation in dampening trophic cascades. Ecol. Lett. 2005, 8, 1299-1306. [CrossRef]

60. Grieshop, M.J.; Flinn, P.W.; Nechols, J.R. Biological control of Indianmeal moth (Lepidoptera: Pyralidae) on finished stored products using egg and larval parasitoids. J. Econ. Entomol. 2006, 99, 1080-1084. [CrossRef]

61. Yamamoto, D.; Henderson, R.; Corley, L.S.; Iwabuchi, K. Intrinsic, inter-specific competition between egg, egg-larval, and larval parasitoids of plusiine loopers. Ecol. Entomol. 2007, 32, 221-228. [CrossRef]

62. Bigler, F.; Babendreier, D.; van Lenteren, J.C. Risk assessment and non-target effects of egg parasitoids in biological control. In Egg Parasitoids in Agroecosystems with Emphasis on Trichogramma; Cônsoli, F.L., Parra, J.R.P., Zucchi, R.A., Eds.; Springer: New York, NY, USA, 2010; pp. 413-442.

63. Strand, M.R.; Vinson, S.B. Facultative hyperparasitism by the egg parasitoid Trichogramma pretiosum (Hymenoptera: Trichogrammatidae). Ann. Entomol. Soc. Am. 1984, 77, 679-686. [CrossRef]

64. Kfir, R.; van Hamburg, H. Interpecific competition between Telenomus ullyetti (Hymenoptera: Scelionidae) and Trichogrammatoidea lutea (Hymenoptera: Trichogrammatidae) parasitizing eggs of the cotton bollworm Heliothis armigera in the laboratory. Environ. Entomol. 1988, 17, 664-670. [CrossRef]

65. Pomari, A.F.; Bueno, A.D.F.; Bueno, R.C.O.D.F.; Menezes-Junior, A.D.O.; Fonseca, A.C.P.F. Releasing number of Telenomus remus (Nixon) (Hymenoptera: Platygastridae) against Spodoptera frugiperda Smith (Lepidoptera: Noctuidae) in corn, cotton and soybean. Ciência Rural 2013, 43, 377-382. [CrossRef] 
66. Jennings, D.E.; Duan, J.J.; Larson, K.M.; Ito, J.P.L.; Shrewsbury, P.M. Evaluating a new method for monitoring the field establishment and parasitism of Oobius agrili (Hymenoptera: Encyrtidae), an egg parasitoid of emerald ash borer (Coleoptera: Buprestidae). Fla. Entomol. 2014, 97, 1263-1265. [CrossRef]

67. Cornelius, M.L.; Dieckhoff, C.; Vinyard, B.T.; Hoelmer, K.A. Parasitism and predation on sentinel egg masses of the brown marmorated stink bug (Hemiptera: Pentatomidae) in three vegetable crops: Importance of dissections for evaluating the impact of native parasitoids on an exotic pest. Environ. Entomol. 2016, 45, 1536-1542. [CrossRef]

68. Jones, A.L.; Jennings, D.E.; Cerruti, R.R.H.; Shrewsbury, P.M. Sentinel eggs underestimate rates of parasitism of the exotic brown marmorated stink bug, Halyomorpha halys. Biol. Control 2014, 78, 61-66. [CrossRef]

69. Nordlund, D.A.; Strand, M.R.; Lewis, W.J.; Vinson, S.B. Role of kairomones from host accessory gland secretion in host recognition by Telenomus remus and Trichogramma pretiosum, with partial characterization. Entomol. Exp. Appl. 1987, 44, 37-43. [CrossRef]

70. Lewis, W.J.; Nordlund, D.A. Semiochemicals influencing fall armyworm parasitoid behavior: Implications for behavioral manipulation. Fla. Entomol. 1984, 67, 343-349. [CrossRef]

71. Penaflor, M.F.G.V.; Erb, M.; Miranda, L.A.; Werneburg, A.G.; Bento, J.M.S. Herbivore-induced plant volatiles can serve as host location cues for a generalist and a specialist egg parasitoid. J. Chem. Ecol. 2011, 37, 1304-1313. [CrossRef]

72. Ballal, C.R.; Jalali, S.K.; Kumar, P. Plant effects on host- parasitoid relations between Spodoptera litura (Fabricius) and Telenomus remus Nixon. Indian J. Plant Prot. 1989, 17, 97-99.

73. Gutierrez-Martinez, A.; Tolon-Becerra, A.; Lastra-Bravo, X.B. Biological control of Spodoptera frugiperda eggs using Telenomus remus Nixon in maize-bean-squash polyculture. Am. J. Agric. Biol. Sci. 2012, 7, 285-292. [CrossRef]

74. Thorpe, K.W. Effects of height and habitat type on egg parasitism by Trichogramma minutum and T. pretiosum (Hymenoptera: Trichogrammatidae). Agric. Ecosyst. Environ. 1985, 12, 117-126. [CrossRef]

75. Andow, D.A.; Prokrym, D.R. Plant structural complexity and host finding by a parasitoid. Oecologia 1990, 82, 162-165. [CrossRef] [PubMed]

76. McCravy, K.W.; Berisford, C.W. Parasitism by Trichogramma spp. (Hymenoptera: Trichogrammatidae) in relation to Nantucket pine tip moth (Lepidoptera: Tortricidae) egg density and location. Environ. Entomol. 1998, 27, 355-359. [CrossRef]

77. Romeis, J.; Shanower, T.G.; Zebitz, C.P.W. Physical and chemical plant characters inhibiting the searching behaviour of Trichogramma chilonis. Entomol. Exp. Appl. 1998, 87, 275-284. [CrossRef]

78. Gingras, D.; Dutilleul, P.; Boivin, G. Effect of plant structure on searching strategy and searching efficiency of Trichogramma turkestanica. J. Insect Sci. 2008, 8, 28. [CrossRef]

79. Pomari, A.F.; Bueno, A.F.; Bueno, R.C.O.F.; Menezes, A.O., Jr. Telenomus remus Nixon egg parasitization of three species of Spodoptera under different temperatures. Neotrop. Entomol. 2013, 42, 399-406. [CrossRef]

80. Vieira, N.F.; Pomari-Fernandes, A.; Lemes, A.A.F.; Vacari, A.M.; De Bortoli, S.A.; Bueno, A.D.F. Cost of production of Telenomus remus (Hymenoptera: Platygastridae) grown in natural and alternative hosts. J. Econ. Entomol. 2017, 110, 2724-2726. [CrossRef]

(C) 2020 by the authors. Licensee MDPI, Basel, Switzerland. This article is an open access article distributed under the terms and conditions of the Creative Commons Attribution (CC BY) license (http://creativecommons.org/licenses/by/4.0/). 